Review

\title{
Generation of Circularly Polarized Luminescence by Symmetry Breaking
}

\author{
Yoshitane Imai \\ Department of Applied Chemistry, Faculty of Science and Engineering, Kindai University, 3-4-1 Kowakae, \\ Higashi-Osaka, Osaka 577-8502, Japan; y-imai@apch.kindai.ac.jp
}

Received: 8 October 2020; Accepted: 23 October 2020; Published: 28 October 2020

\begin{abstract}
Circularly polarized luminescence (CPL) has attracted significant attention in the fields of chiral photonic science and optoelectronic materials science. In a CPL-emitting system, a chiral luminophore derived from chiral molecules is usually essential. In this review, three non-classical CPL (NC-CPL) systems that do not use enantiomerically pure molecules are reported: (i) supramolecular organic luminophores composed of achiral organic molecules that can emit CPL without the use of any chiral auxiliaries, (ii) achiral or racemic luminophores that can emit magnetic CPL (MCPL) by applying an external magnetic field of $1.6 \mathrm{~T}$, and (iii) circular dichroism-silent organic luminophores that can emit CPL in the photoexcited state as a cryptochiral CPL system.
\end{abstract}

Keywords: chiral; circularly polarized luminescence (CPL); magnetic circularly polarized luminescence (MCPL); spontaneous resolution

\section{Introduction}

The potential application of luminescent techniques to various systems, such as organic and organometallic electroluminescence devices and optoelectronic devices, has attracted considerable attention [1-6]. Analogous to the chirality associated with molecules, there exists chirality of light, which is referred to as circularly polarized luminescence (CPL). Unlike circular dichroism (CD), which indicates the chirality of the ground state, CPL spectroscopy elucidates conformational and structural information pertaining to optically active molecules in the photoexcited state. Optically active luminescent materials may produce either clockwise or anti-clockwise CPL. Chiral luminophores demonstrating CPL have attracted research attention, particularly in the fields of chiral photonic science and optoelectronic materials science [7-18].

CPL generally requires chiral organic or organometallic luminophores. In organometallic luminophores, chiral organic ligands coordinating with optically active metal ions induce chirality in the luminescent complex. In such a CPL-emitting system, a chiral organic molecule is indispensable. In addition, in practical applications of CPL, both right- and left-handed CPL are used, and their selective emission requires chiral organic or organometallic luminophores with opposite chirality. Chiral organic luminophores that can be prepared from achiral or racemic molecules are preferred as efficient and industrially useful chiral luminophores $[19,20]$.

In this review, three types of non-classical CPL (NC-CPL) systems, including symmetry breaking CPL (SB-CPL) systems, are reported. The first is a spontaneous-resolution CPL system using achiral or racemic molecules. The second is a magnetic circularly polarized luminescence (MCPL) system based on achiral or racemic molecules under an external magnetic field. Finally, a cryptochiral CPL system based on CD-silent molecules in the photoexcited state is discussed. 


\section{CD and CPL from Achiral and Racemic Molecules by Spontaneous Resolution}

Chiral organic luminophores are typically prepared via several steps from chiral molecules. Unfortunately, few chiral starting molecules are readily available. In addition, commercial chiral molecules are generally more expensive than achiral or racemic molecules. To eliminate such concerns, it would be economically and industrially highly useful if a chiral organic luminophore could be produced via an achiral or racemic compound.

The origin and amplification of chirality, which has led to an overwhelming enantio-enrichment of organic molecules on Earth, has been a significant topic of interest in this field of science for many decades. One proposed hypothesis for the origin of chirality is the spontaneous resolution of one of the two possible enantiomers of chiral crystals from achiral or racemic molecules [21-30]. For example, 2-anthracenecarboxylic acid (1) is an achiral luminescent molecule, and racemic 1-phenylethylamine $(\mathrm{rac}-2)$ is a racemic molecule (an equimolar mixture of $(R)$ - and (S)-1-phenylethylamine) (Figure 1 ). When a mixture of $\mathbf{1}$ and rac-2 is crystallized from solution, the chiral supramolecular organic luminophores I or $\mathbf{I}^{\prime}$ can be preferentially obtained by spontaneous resolution. Chiral luminophores $\mathbf{I}$ and $\mathbf{I}^{\prime}$ are an enantiomeric pair and are composed of $\mathbf{1}$ and (R)-2 for $\mathbf{I}$, and $\mathbf{1}$ and (S)-2 for $\mathbf{I}^{\prime}$. $\mathbf{I}$ or $\mathbf{I}^{\prime}$ can be obtained selectively by using the corresponding seed crystals.

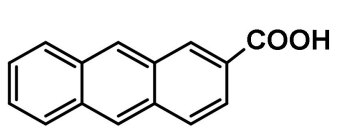

1

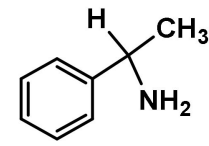

rac-2

Figure 1. Achiral and racemic component molecules for chiral organic luminophores.

Unlike many organic luminophores, chiral luminophore $\mathbf{I}$ (or $\mathbf{I}^{\prime}$ ) is able to emit luminescence even in the solid state without aggregate quenching. The solid-state luminescences of $\mathbf{I}$ and $\mathbf{I}^{\prime}$ are shifted to a shorter wavelength (by $34 \mathrm{~nm}$ ) relative to that of their component luminescent molecule 1 . The solid-state luminescent maximum $\left(\lambda_{\mathrm{PL}}\right)$ of $\mathbf{I}\left(\right.$ or $\left.\mathbf{I}^{\prime}\right)$ is $430 \mathrm{~nm}$. The photoluminescence quantum yield $\left(\Phi_{\mathrm{F}}\right)$ increases from $4 \%$ for $\mathbf{1}$ to $20 \%$ for I. The solid-state CD spectra of I (indicated by the red line) and $\mathbf{I}^{\prime}$ (indicated by the blue line) are mirror images (Figure 2). The CD signals derived from the fluorescent anthracene unit clearly appear between 330 and $450 \mathrm{~nm}$. The circular anisotropy factor $\left(g_{\mathrm{CD}}\right)$ of the major $\mathrm{CD}$ Cotton band $\left(\lambda_{\mathrm{CD}}=404 \mathrm{~nm}\right)$ of $\mathbf{I}$ is approximately $-0.6 \times 10^{-3}$. This shows that the fluorescent anthracene units effectively exist in a chiral environment in the solid state.

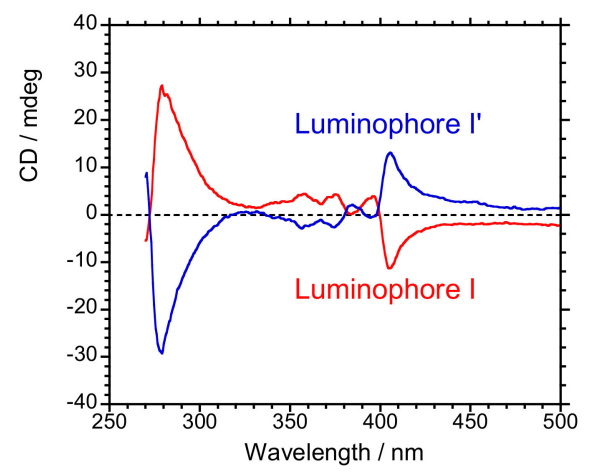

Figure 2. Solid-state circular dichroism (CD) spectra of luminophores I (red line) and $\mathbf{I}^{\prime}$ (blue line) in the solid state (KBr pellets).

As expected, $\mathrm{X}$-ray analysis shows that luminophore $\mathbf{I}$ has a $P 2_{1}$ chiral space group and a characteristic 21 -helical network column (Figure 3a). This characteristic column is mainly formed via ionic and hydrogen bonds composed of the carboxylic acid anions of $\mathbf{1}$ and the protonated amine 
cations of $(R)-\mathbf{2}(\mathbf{1}:(R)-\mathbf{2}=1: 1$ component ratio). Luminophore $\mathbf{I}$ is constructed via the aggregation of these 2 -helical network columns by three types of edge-to-face interactions: anthracene-anthracene edge-to-face, benzene-anthracene edge-to-face, and anthracene-benzene edge-to-face interactions (Figure $3 b$ ). This suggests that the formation of a $2{ }_{1}$-helical network column is a key factor in the production of spontaneously resolved chiral organic luminophores [31].

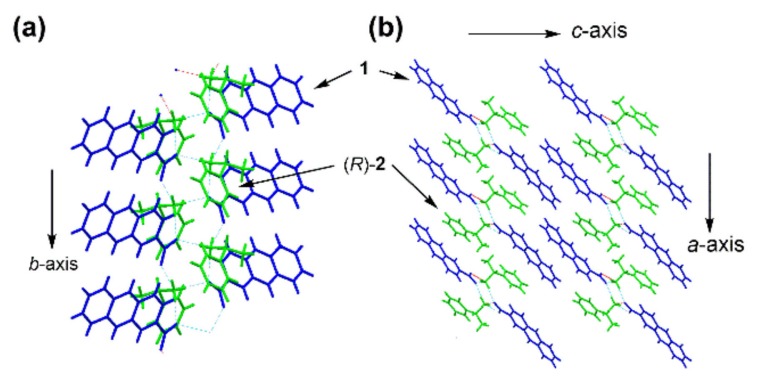

Figure 3. Crystal structures of luminophore I. (a) 21 -Helical columnar network structure along the $b$-axis. (b) Packing structure observed along the $b$-axis.

Chiral organic luminophore I (or I') was successfully prepared by combining an achiral fluorescent molecule and a racemic molecule as a spontaneous-resolution system. Thus, by using only achiral molecules, chiral organic luminophores were formed.

When a mixture of the achiral luminescent molecule 2-anthracenecarboxylic acid (1) and the achiral molecule benzylamine (3) (Figure 4) is crystallized from solution at room temperature, spontaneous resolution results in the chiral supramolecular organic luminophore II or II', which are composed of $\mathbf{1}$ and 3 with opposite chirality. In this case, a small amount of III, another polymorphic luminophore, is also obtained. Luminophores II or II' can also be selectively obtained by using the corresponding seed crystals.

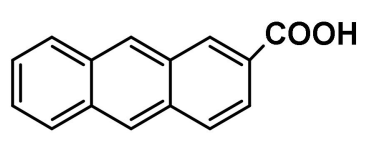

1<smiles>NCc1ccccc1</smiles>

3

Figure 4. Achiral component molecules for circularly polarized luminescence (CPL).

As expected, chiral luminophore II also has an anion-cation and hydrogen bonded $2{ }_{1}$-helical network column, similar to that of the spontaneously resolved chiral luminophore I (Figure 5a). The stoichiometry of components $\mathbf{1}$ and $\mathbf{3}$ in luminophore II is 1:1, and the space group is chiral $\left(P 2_{1}\right)$. Each network column is held in place through two types of edge-to-face interactions: anthracene-anthracene edge-to-face and benzene-anthracene edge-to-face interactions. The luminophore is created by the aggregation of this network column (Figure 5b).

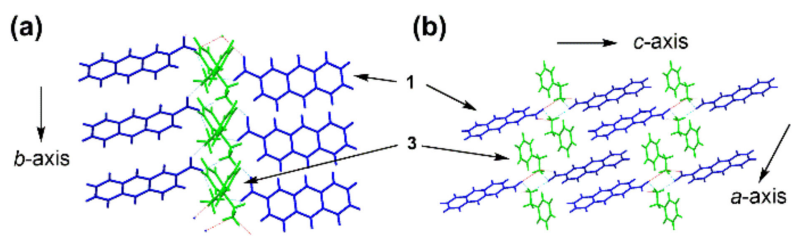

Figure 5. Crystal structures of luminophore II. (a) $2{ }_{1}$-Helical columnar network along the $b$-axis. (b) Packing structure observed along the $b$-axis. 
Chiral luminophores II and II' show CD in the solid state (Figure 6). Characteristic mirror-image CD spectra of II and II' are observed with a major peak around $416 \mathrm{~nm}$ (Figure 6). This peak originated from the fluorescent anthracene unit. The $g_{\mathrm{CD}}$ value of the major CD Cotton band $\left(\lambda_{\mathrm{CD}}=416 \mathrm{~nm}\right)$ of II is approximately $-1.0 \times 10^{-3}$.

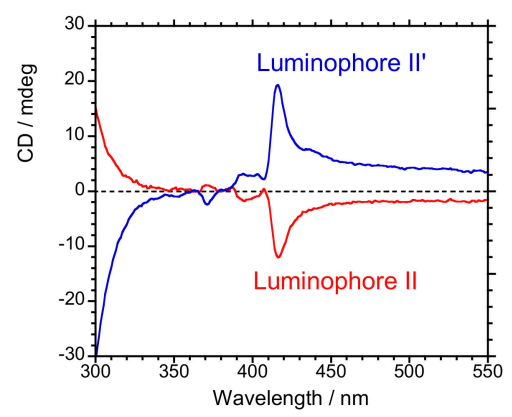

Figure 6. Solid-state CD spectra of luminophores II (red line) and II' (blue line) in the solid state (KBr pellets).

Luminophore II exhibits luminescence with a solid-state luminescent maximum $\left(\lambda_{\mathrm{PL}}\right)$ of $446 \mathrm{~nm}$ and a photoluminescence quantum yield $\left(\Phi_{\mathrm{F}}\right)$ of $16 \%$, which is four times greater than that of the component luminophore $\mathbf{1}$ in the solid state. Interestingly, II, having a negative Cotton CD band, emits negative CPL with a circular anisotropy factor (Kuhn's dissymmetry ratio: $g_{\mathrm{CPL}}$ ) of approximately $-1.1 \times 10^{-3}$, even though II is composed of achiral molecules (Figure 7). It can be concluded from the crystal structure and the theory of oscillator coupling that the CPL originates from the inter-columnar anthracene units between adjoining $2{ }_{1}$-helical columns.

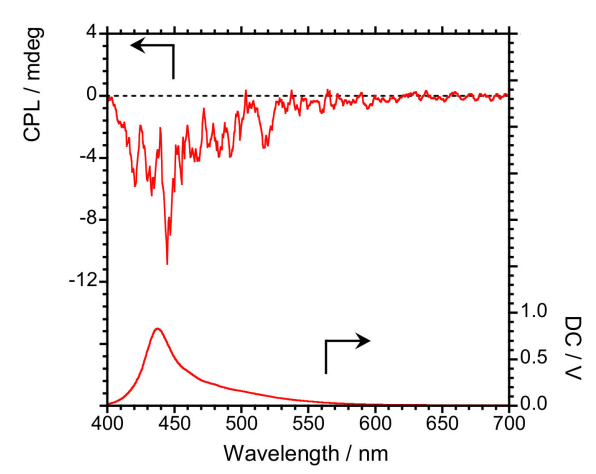

Figure 7. Solid-state CPL and the unpolarized photoluminescence (PL) spectra of luminophore II in the solid state (KBr pellet).

In this system, the production of a small amount of polymorphic luminophore III is an issue. Notably, this problem can be solved by changing the crystallization method. When solid-state luminescent molecule $\mathbf{1}$ is left to stand in the vapor of liquid molecule $\mathbf{3}$ at room temperature, only III is selectively obtained. In contrast, when solid $\mathbf{1}$ and liquid $\mathbf{3}$ are directly mixed and ground using an agate mortar, only chiral II or II' is produced.

Because many chiral molecules are not easily available and chiral molecules are more expensive than achiral or racemic molecules, chiral organic luminophores prepared from achiral or racemic molecules are preferred as industrial chiral luminophores. This study provides useful information for the creation of new spontaneously resolved CPL luminescent systems without using chiral factors [32]. 


\section{CPL from Optically Inactive Organometallic and Organic Luminophores under a Magnetic Field}

Organometallic luminophores have been a focus of CPL materials. Optically active lanthanide luminophores coordinated with chiral organic ligands in particular show an extremely narrow full-width at half-maximum CPL, with a high circular anisotropy factor from the visible to near-infrared regions [33-38]. In order to impart CPL characteristics to lanthanide luminophores, a chirality-inducible ligand causing antenna effects through ligand-to-metal charge transfer is inevitably needed. In recent years, an external static magnetic field has been used to act as a versatile chirality-inducible physical force in the ground and excited states, and to perturb chiral electronic structures toward several optically inactive and achiral luminophores [39-46]. Such an external magnetic influence at the molecular level causes the optically inactive luminophore to emit MCPL. In this section, the MCPL capabilities of optically inactive lanthanide luminophores $\mathrm{Eu}(\mathrm{III})(\mathrm{hfa})_{3}$ and $\mathrm{Tb}(\mathrm{III})(\mathrm{hfa})_{3}$ (Figure 8) are discussed.
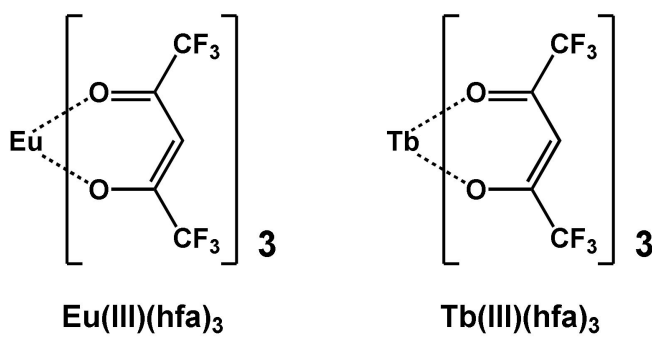

Figure 8. Optically inactive $\mathrm{Eu}(\mathrm{III})(\mathrm{hfa})_{3}$ and $\mathrm{Tb}(\mathrm{III})(\mathrm{hfa})_{3}$.

CPL was not observed for $\mathrm{Eu}(\mathrm{III})(\mathrm{hfa})_{3}$ in the absence of an external magnetic field. In contrast, $\mathrm{Eu}(\mathrm{III})(\mathrm{hfa})_{3}$ can emit MCPL and magnetic-field-induced unpolarized photoluminescence (hereafter denoted as $\mathrm{PL}$, rather than MCPL) in $\mathrm{CHCl}_{3}$ and acetone solutions under a $1.6 \mathrm{~T}$ magnetic field, as shown in Figure 9 (blue lines for $\mathrm{CHCl}_{3}$ and red lines for acetone). The spectra under the $\mathrm{N} \rightarrow \mathrm{S}$ (N-up) magnetic field along the direction of the excitation light are shown via solid lines, and the spectra under the $\mathrm{S} \rightarrow \mathrm{N}$ (S-up) magnetic field along the direction of the excitation light are shown via dotted lines.

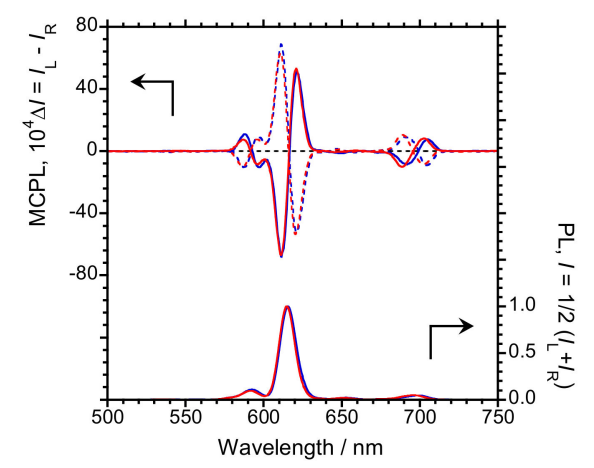

Figure 9. Magnetic circularly polarized luminescence (MCPL) (upper panel) and magnetic-field-induced unpolarized photoluminescence (PL) (lower panel) spectra of $\mathrm{Eu}(\mathrm{III})(\mathrm{hfa})_{3}$ in $\mathrm{N} \rightarrow \mathrm{S}$ (solid lines) and $\mathrm{S} \rightarrow \mathrm{N}$ (dotted lines) configurations under a $1.6 \mathrm{~T}$ magnetic field in $\mathrm{CHCl}_{3}$ (blue lines) and acetone (red lines) solutions $\left(1.0 \times 10^{-3} \mathrm{M}\right)$.

The MCPL spectra (upper panel in Figure 9) of $\mathrm{Eu}(\mathrm{III})(\mathrm{hfa})_{3}$ in the two solutions are similar. The meaningful MCPL peaks $\left(\lambda_{\mathrm{MCPL}}\right)$ observed were $\approx 587,596,611,621,690$ and $703 \mathrm{~nm}$. They correspond to the $4 \mathrm{f}-4 \mathrm{f}$ transitions of $\mathrm{Eu}(\mathrm{III})$. The $\mathrm{N} \rightarrow \mathrm{S}$ magnetic field MCPL spectrum and $\mathrm{S} \rightarrow \mathrm{N}$ magnetic field MCPL spectrum are almost mirror images of each other. They show that the MCPL sign can be attributed to the direction of applied external magnetic field $(\mathrm{N} \rightarrow \mathrm{S}$ or $\mathrm{S} \rightarrow \mathrm{N})$. 
To discuss the quantitative MCPL efficiency, the $g_{\mathrm{MCPL}}$ values are used. The $g_{\mathrm{MCPL}}$ factor is defined as $g_{\mathrm{MCPL}}=2\left(I_{\mathrm{L}}-I_{\mathrm{R}}\right) /\left(I_{\mathrm{L}}+I_{\mathrm{R}}\right)$, and normalized by an external magnetic field $\left(\mathrm{T}^{-1}\right)$. In this equation, $I_{\mathrm{L}}$ and $I_{\mathrm{R}}$ show the left- and right-handed apparent MCPL intensities, respectively, when photoexcited by unpolarized light. The $\left|g_{\mathrm{MCPL}}\right|$ values of the main bands in $\mathrm{CHCl}_{3}$ were $0.81 \times 10^{-2} \mathrm{~T}^{-1}$ at $597 \mathrm{~nm}$ and $0.63 \times 10^{-2} \mathrm{~T}^{-1}$ at $611 \mathrm{~nm}$. The $\left|g_{\mathrm{MCPL}}\right|$ values of the two solutions are similar. These results indicate that the external static magnetic field can clearly induce $\mathrm{CPL}$ from optically inactive $\mathrm{Eu}(\mathrm{III})(\mathrm{hfa})_{3}$, or possibly a mixture of the $\Delta$ - and $\Lambda$-forms of $D_{3}$-symmetric $\mathrm{Eu}(\mathrm{III})(\mathrm{hfa})_{3}$, in the solution state. The MCPL of $\mathrm{Eu}(\mathrm{III})(\mathrm{hfa})_{3}$ was further investigated in three types of solid states-polymethylmethacrylate (PMMA) film, $\mathrm{KBr}$ pellet, and powder-under the same 1.6 T magnetic field as was used for the solution state measurements. The MCPL emitted from these solid-state luminophores was practically the same as that from the solution state.

Similarly, the $1.6 \mathrm{~T}$ magnetic field induces CPL in optically inactive $\mathrm{Tb}(\mathrm{III})(\mathrm{hfa})_{3}$ luminophores, or possibly a mixture of the $\Delta$ - and $\Lambda$-forms of $D_{3}$-symmetric $\mathrm{Tb}$ (III) luminophores, in $\mathrm{CHCl}_{3}$ and acetone solutions. The $\mathrm{N} \rightarrow \mathrm{S}$ and $\mathrm{S} \rightarrow \mathrm{N}$ magnetic fields can control the sign of the MCPL of the $\mathrm{Tb}$ (III) luminophores, as shown in Figure 10 (blue lines for $\mathrm{CHCl}_{3}$ and red lines for acetone; solid lines for $\mathrm{N} \rightarrow \mathrm{S}$ and dotted lines for $\mathrm{S} \rightarrow \mathrm{N}$ configurations under 1.6 T). The $\lambda_{\mathrm{MCPL}}$ maxima and the $\left|g_{\mathrm{MCPL}}\right|$ values of $\mathrm{Tb}(\mathrm{III})(\mathrm{hfa})_{3}$ in $\mathrm{CHCl}_{3}$ are $0.53 \times 10^{-2} \mathrm{~T}^{-1}$ at $484 \mathrm{~nm}, 0.46 \times 10^{-2} \mathrm{~T}^{-1}$ at $493 \mathrm{~nm}, 0.16 \times 10^{-2} \mathrm{~T}^{-1}$ at $538 \mathrm{~nm}$, and $0.094 \times 10^{-2} \mathrm{~T}^{-1}$ at $553 \mathrm{~nm}$, which correspond to the characteristic $4 \mathrm{f}-4 \mathrm{f}$ transitions of $\mathrm{Tb}(\mathrm{III})$ in $\mathrm{CHCl}_{3}$. The $\left|g_{\mathrm{MCPL}}\right|$ values of the $\mathrm{CHCl}_{3}$ and acetone solutions are similar.

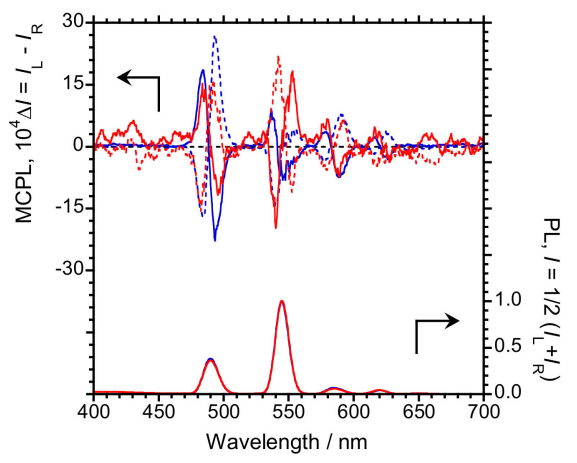

Figure 10. MCPL (upper panel) and PL (lower panel) spectra of $\mathrm{Tb}(\mathrm{III})(\mathrm{hfa})_{3}$ in $\mathrm{N} \rightarrow \mathrm{S}$ (solid lines) and $\mathrm{S} \rightarrow \mathrm{N}$ (dotted lines) magnetic directions under a $1.6 \mathrm{~T}$ magnetic field in $\mathrm{CHCl}_{3}$ (blue lines) and acetone (red lines) solutions $\left(1.0 \times 10^{-3} \mathrm{M}\right)$.

Interestingly, the signs of the MCPL spectra of $\mathrm{Tb}(\mathrm{III})(\mathrm{hfa})_{3}$ between the $\mathrm{CHCl}_{3}$ and acetone solutions are partially inverted in the $\mathrm{N} \rightarrow \mathrm{S}$ and $\mathrm{S} \rightarrow \mathrm{N}$ magnetic fields. In $\mathrm{CHCl}_{3}$, the $\mathrm{N} \rightarrow \mathrm{S}$ magnetic field spectrum clearly shows positive(+)-/negative(-)-sign MCPL signals at 538/553 nm, derived from the ${ }^{5} \mathrm{D}_{4} \rightarrow{ }^{7} \mathrm{~F}_{5}$ transitions. In contrast, the signs of the MCPL signals derived from the same ${ }^{5} \mathrm{D}_{4} \rightarrow{ }^{7} \mathrm{~F}_{5}$ transitions are reversed in acetone, and are clearly negative(-)-/positive(+)-sign MCPL. This sign inversion may be caused by the different coordination environment around $\mathrm{Tb}$ (III) of lone pair electrons on the $\mathrm{C}=\mathrm{O}$ oxygen of acetone. It appears from these results that the direction of rotation of the MCPL from $\mathrm{Tb}(\mathrm{III})(\mathrm{hfa})_{3}$ can be controlled by both the magnetic field direction and the nature of the solvent molecule. Similar to the chiroptical characteristics of $\mathrm{Eu}(\mathrm{III})(\mathrm{hfa})_{3}, \mathrm{MCPL}$ from Tb(III)(hfa) ${ }_{3}$ can also be emitted in solid states, such as a PMMA film, KBr pellet and powder, under a 1.6 T magnetic field [47].

Optically active $\pi$-conjugated organic luminophores demonstrating CPL are attracting attention in the fields of chiral photonic and optoelectronic materials science. Most organic CPL luminophores are prepared from chiral organic starting materials. Here, we describe CPL emitted from an achiral organic luminophore under an external magnetic field. Three typical $\pi$-conjugated luminophores-pyrene (Py), 1-pyrenol (1-PyOH) and 2-pyrenol (2-PyOH) —which do not possess any chiral sources were investigated (Figure 11). 

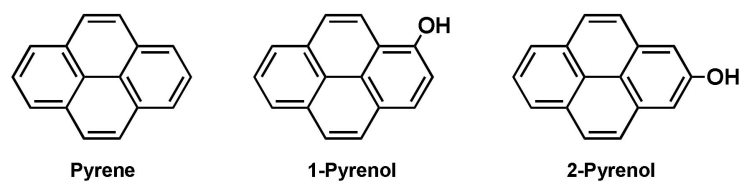

Figure 11. Achiral luminophores Py, 1-PyOH, and 2-PyOH.

Surprisingly, these achiral pyrene luminophores clearly emit mirror-image MCPLs under a $1.6 \mathrm{~T}$ magnetic field in $\mathrm{CHCl}_{3}$ solution when Faraday-type $\mathrm{N} \rightarrow \mathrm{S}$ or $\mathrm{S} \rightarrow \mathrm{N}$ geometry is employed, similarly to lanthanoid luminophores, as shown in Figure 12 (solid lines for the $\mathrm{N} \rightarrow \mathrm{S}$ magnetic field and dotted lines for the $\mathrm{S} \rightarrow \mathrm{N}$ magnetic field). In addition, the signs of the MCPL can be controlled by the position (1- or 2-position) of the $\mathrm{OH}$ group on the peripheral pyrene ring under the same $\mathrm{N} \rightarrow \mathrm{S}$ or $\mathrm{S} \rightarrow \mathrm{N}$ geometry.

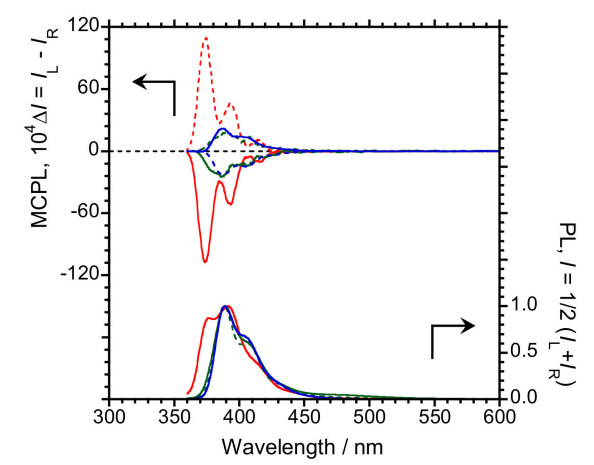

Figure 12. MCPL (upper panel) and PL (lower panel) spectra of Py (red lines), 1-PyOH (green lines), and 2-PyOH (blue lines) in $\mathrm{N} \rightarrow \mathrm{S}$ (solid lines) and $\mathrm{S} \rightarrow \mathrm{N}$ (dotted lines) magnetic directions under a $1.6 \mathrm{~T}$ magnetic field in $\mathrm{CHCl}_{3}$ solution $\left(1.0 \times 10^{-4} \mathrm{M}\right)$.

In Py, luminescence peaks $\left(\lambda_{\mathrm{MCPL}}\right)$ can be observed at 374, 393 and $416 \mathrm{~nm}$. These correspond to the $0-0^{\prime}, 0-1^{\prime}$ and $0-2^{\prime}$ vibronic PL bands of the pyrenyl monomer species. The luminescence peaks of 1-PyOH and 2-PyOH are similar to those of Py. The $\left|g_{\mathrm{MCPL}}\right|$ value of $\mathbf{P y}$ in $\mathrm{CHCl}_{3}$ is $0.82 \times 10^{-3} \mathrm{~T}^{-1}$ at $374 \mathrm{~nm}$. Pyrene has monomer and excimer luminescence. In the three pyrenes, the pyrenyl excimer-origin MCPL cannot be detected around 450-500 nm even at the high concentration of $1.0 \times 10^{-2} \mathrm{M}$. This indicates that pyrene excimers in the excited state apparently do not contribute to MCPL.

By using this method, various magnetic circularly polarized luminophores can be prepared from optically inactive and achiral molecules. In the future, various achiral luminophores sandwiched by two permanent magnetic fields should be able to emit MCPL from the visible to near-infrared wavelength regions, and the sign of the MCPL could be modulated by the alternating current magnetic field [48].

\section{CPL from Cryptochiral Organic Luminophores}

In 1977, Mislow reported on cryptochirality $[49,50]$, which means hidden molecular chirality. This novel and unique chiroptical phenomenon contributes to molecular cryptography, steganography and watermarks. The most reported chiral CPL luminophores are CD-active/CPL-active luminophores. To overcome this limitation, [(4R,5R)-2,2-dimethyl-1,3-dioxolane-4,5-diyl]bis-methanolyl-bis-1-pyrene $[(R, R)-4]$ and its enantiomer $[(S, S)-4]$ were designed (Figure 13). These luminophores can be prepared from the corresponding enantiomerically pure 2,3-O-isopropylidene-threitol and 1-pyreneacetic acid in a single step. 


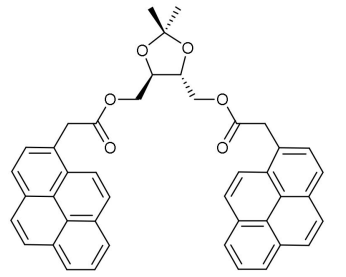

$(R, R)-4$

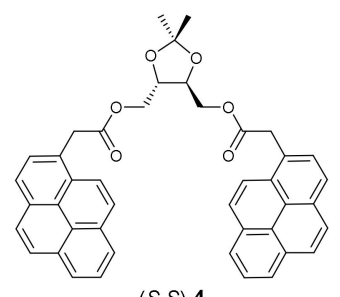

$(S, S)-4$

Figure 13. Cryptochiral dioxolane-bipyrene luminophores $(R, R)-4$ and $(S, S)-4$.

A characteristic of these luminophores is that the chiral 2,2-dimethyl-1,3-dioxolane unit acts as a chiral inducer and conductor to induce chirality in the two distant fluorescent pyrene moieties. The two pyrenes are far from the central chirality of the 2,2-dimethyl-1,3-dioxolane unit and act as a hidden chiral excimer derived from photoexcitation, which is the heart of cryptochirality. Under the influence of this conductor, 4 can induce a preferential chiral twist of the fluorescent pyrenes upon an external bias on demand.

As shown in the lower panel of Figure 14, the UV-vis absorption spectrum of $(R, R)-4$ (red line) (or $(S, S)-4$ (blue line)) shows two major well-resolved vibronic $\pi-\pi^{*}$ transitions of isolated pyrene in the 250-290 and 300-370 $\mathrm{nm}$ regions in $\mathrm{CHCl}_{3}$ solution. However, $(R, R)-\mathbf{4}($ or $(S, S)-\mathbf{4})$ does not provide any meaningful CD signals (Figure 14, upper panel). In contrast, $(R, R)-4$ (red line) (or $(S, S)-4$ (blue line)) can clearly emit CPL at $460 \mathrm{~nm}$ with an $18 \% \Phi_{\mathrm{F}}$ value, originating from the excimer pyrene in $\mathrm{CHCl}_{3}$ solution (Figure 15, upper panel). Luminophores $(R, R)-4$ and $(S, S)-4$ provide nearly mirror-image CPL spectra. Their $\left|g_{\mathrm{CPL}}\right|$ value is $\sim 8.9 \times 10^{-4}$ at approximately $460 \mathrm{~nm}$ in $\mathrm{CHCl}_{3}$.

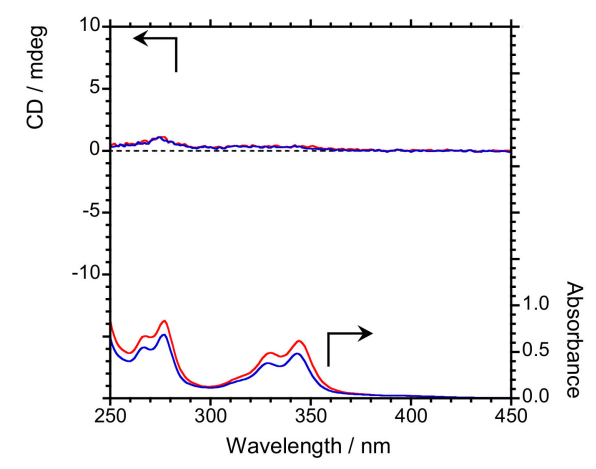

Figure 14. CD (upper panel) and UV-vis absorption (lower panel) spectra of $(R, R)-4$ (red lines) and $(S, S)-4$ (blue lines) in $\mathrm{CHCl}_{3}$ solution $\left(1.0 \times 10^{-4} \mathrm{M}\right)$.

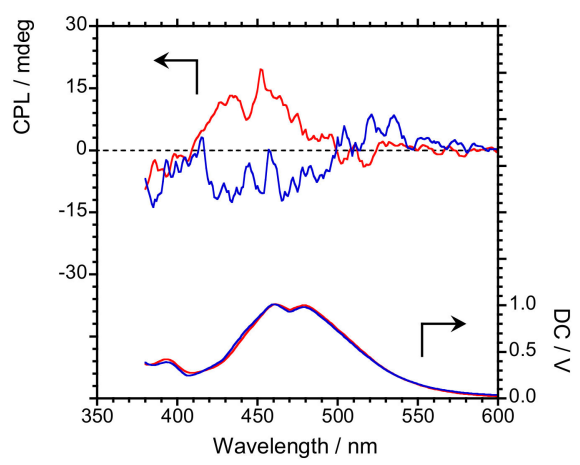

Figure 15. CPL (upper panel) and PL (lower panel) spectra of $(R, R)-4$ (red lines) and $(S, S)-4$ (blue lines) in $\mathrm{CHCl}_{3}$ solution $\left(1.0 \times 10^{-4} \mathrm{M}\right)$. 
The most probable structures of $(R, R)-4$ in both the ground and photoexcited states were calculated. From these calculations, a model of this mechanism was developed and is shown in Figure 16. The structure obtained for the ground state does not adopt face-to-face pyrenyl $\pi-\pi$ stacking conformers or slip pyrenyl $\pi-\pi$ dimer conformers, but adopts the T-shaped conformers of pyrenes. These unique pyrene conformations may be the reason for the nonexistent or weak CD signals in the ground state. In contrast, $(R, R)-4$ adopts chiral pyrenyl $\pi-\pi$-stacked conformers in the photoexcited state. Thus, the CPL magnitudes may be enhanced by the chiral $\pi-\pi$ pyrenyl stacks, providing chiroptically detectable signals [51].
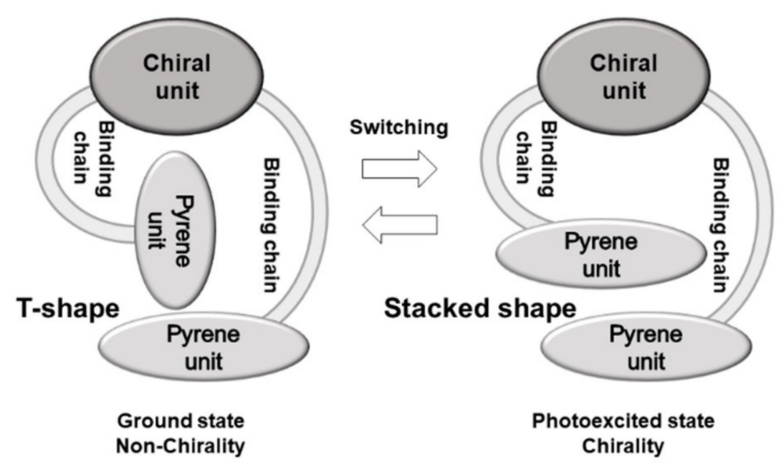

Figure 16. Mechanism of cryptochiral CPL system.

Based on this mechanism, instead of using a central chiral 2,2-dimethyl-1,3-dioxolane unit that acts as a controller for the expression of chirality of two pyrenes, a cryptochiral CD-silent/CPL-active luminophore was designed using an axially chiral binaphthyl unit. Chiral luminophores $(R)-5$ and (S)-5 are composed of the corresponding chiral 5,5',6,6',7,7',8, $8^{\prime}$-octahydro-1,1'-bi-2-naphthyl as the chiral unit, and two pyrenes as fluorescent units connected through flexible ester tethers (Figure 17).

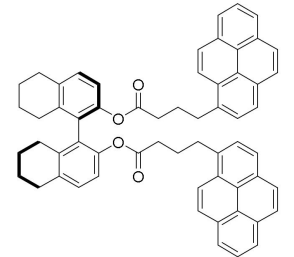

$(R)-5$

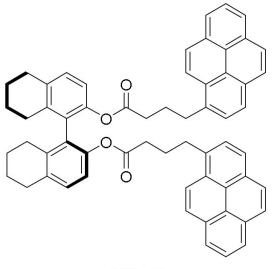

(S)-5

Figure 17. Cryptochiral binaphthyl-bipyrene luminophores (R)-5 and (S)-5.

To study the ground state chiroptical properties of 5, the CD and UV-vis absorption spectra of $(R)-5$ and (S)-5 were measured, in the same manner as 4 (Figure 18). In the UV-vis absorption spectra, three main $\pi-\pi^{*}$ vibronic transitions $\left({ }^{1} \mathrm{~L}_{\mathrm{a}}\right.$ transitions) derived from two pyrene moieties were observed between 315 and $360 \mathrm{~nm}$. On the other hand, the intensities of the CD signals of 5, corresponding to these UV bands, were noticeably weak.

The PL and CPL spectra of (R)-5 and (S)-5 in $\mathrm{CHCl}_{3}$ are shown in Figure 19. From luminophore 5, strong excimer PL is emitted at $468 \mathrm{~nm}\left(\lambda_{\mathrm{PL}}\right)$ from the pyrenes. In addition, luminophores $(R)-5$ and (S)-5 emit clear CPL, and each CPL sign is positive and negative, respectively. The absolute $g_{\mathrm{CPL}}$ value for $(R)-5$ is $+2.5 \times 10^{-3}$ at $454 \mathrm{~nm}$. This suggests that because of the moderately controlled flexible framework, chiral luminophore 5 functions as a cryptochiral CPL system whose CD properties cannot be detected. 


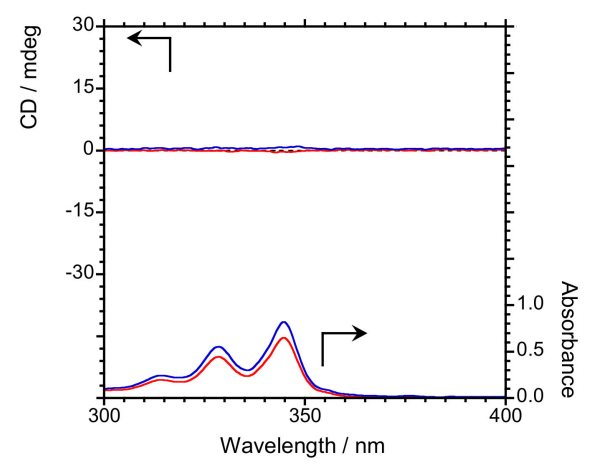

Figure 18. CD (upper panel) and UV-vis absorption (lower panel) spectra of (R)-5 (red lines) and (S)-5 (blue lines) in $\mathrm{CHCl}_{3}$ solution $\left(1.0 \times 10^{-5} \mathrm{M}\right)$.

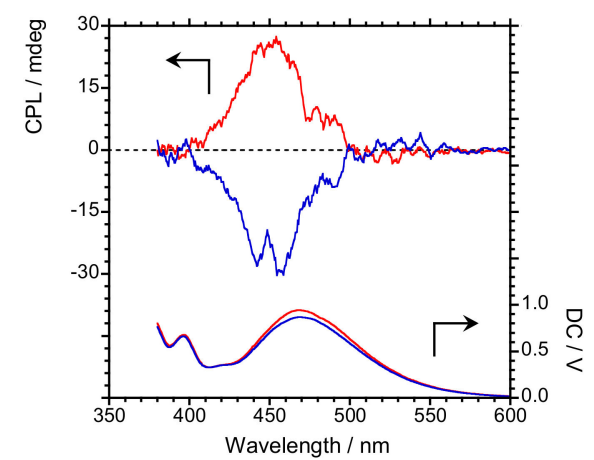

Figure 19. CPL (upper panel) and PL (lower panel) spectra of (R)-5 (red lines) and (S)-5 (blue lines) in $\mathrm{CHCl}_{3}$ solution $\left(1.0 \times 10^{-5} \mathrm{M}\right)$.

This cryptochiral CPL system is similar to the cryptochiral dioxolane-bipyrene luminescent system 4 . The system simultaneously controls two chiral points (binaphthyl and pyrene units) in the photoexcited state through suitable choices of rotamer, linkers and fluorophores. In luminophore 5, the two fluorescent pyrene units are in the conformation of an almost achiral T-shape in the ground state. Moreover, the $\theta$ value of the axially chiral octahydrobinaphthyl unit is approximately $80^{\circ}-90^{\circ}$. The unique arrangements and conformations of the two pyrene and one binaphthyl units make the CD spectrum almost undetectable [52]. In the photoexcited state, however, the conformation of two pyrene units changes to a chiral $\pi$-stacked spatial arrangement. These configuration transfers are responsible for the cryptochiral properties, meaning the silent $C D$ and active CPL derived from the excimer pyrene moiety [53].

\section{Conclusions}

In most CPL-emitting systems, a chiral luminophore derived from chiral molecules is essential. In addition, the selective emission of right- and left-handed CPL requires chiral organic or organometallic luminophores with opposite chirality. However, non-classical CPL (NC-CPL) systems that use no enantiomerically pure molecules have been reported. Such systems include: (i) a spontaneous-resolution CPL system, in which supramolecular organic luminophores prepared from achiral organic molecules can emit CPL without the use of any chiral auxiliaries; (ii) achiral or racemic luminophores that can emit CPL, viz. MCPL, by applying a $1.6 \mathrm{~T}$ external magnetic field in both the solution and solid states; and (iii) a cryptochiral CPL system, in which CD-silent organic luminophores can emit CPL in the photoexcited state. These systems indicate that CPL can be emitted from achiral or racemic molecules by suitable symmetry breaking. 
Funding: This review was supported by a Grant-in-Aid for Scientific Research (nos. 18K05094, 19H02712, 19H04600, and 20H04678) from the MEXT/Japan Society for the Promotion of Science and the Research Foundation. This review was also supported by JST, CREST (JPMJCR2001), Japan.

Acknowledgments: Y.I. thanks Michiya Fujiki (Nara Institute of Science and Technology) and Reiko Kuroda (Chubu University) for helpful discussions regarding the origin of circularly polarized luminescence phenomena.

Conflicts of Interest: The authors declares no conflict of interest.

\section{References}

1. Yang, X.; Zhou, G.; Wong, W.Y. Functionalization of phosphorescent emitters and their host materials by main-group elements for phosphorescent organic light-emitting devices. Chem. Soc. Rev. 2015, 44, 8484-8575. [PubMed]

2. Veldhuis, S.A.; Boix, P.P.; Yantara, N.; Li, M.; Sum, T.C.; Mathews, N.; Mhaisalkar, S.G. Perovskite Materials for Light-Emitting Diodes and Lasers. Adv. Mater. 2016, 28, 6804-6834. [PubMed]

3. Im, Y.; Kim, M.; Cho, Y.J.; Seo, J.; Yook, K.S.; Lee, J.Y. Molecular Design Strategy of Organic Thermally Activated Delayed Fluorescence Emitters. Chem. Mater. 2017, 29, 1946-1963. [CrossRef]

4. Huang, T.; Jiang, W.; Duan, L. Recent progress in solution processable TADF materials for organic light-emitting diodes. J. Mater. Chem. C 2018, 6, 5577-5596.

5. Ma, X.; Wang, J.; Tian, H. Assembling-Induced Emission: An Efficient Approach for Amorphous Metal-Free Organic Emitting Materials with Room-Temperature Phosphorescence. Acc. Chem. Res. 2019, 52, 738-748.

6. Zhang, D.-W.; Li, M.; Chen, C.-F. Recent advances in circularly polarized electroluminescence based on organic light-emitting diodes. Chem. Soc. Rev. 2020, 49, 1331-1343.

7. Field, J.E.; Muller, G.; Riehl, J.P.; Venkataraman, D. Circularly Polarized Luminescence from Bridged Triarylamine Helicenes. J. Am. Chem. Soc. 2003, 125, 11808-11809.

8. Maeda, H.; Bando, Y. Recent progress in research on stimuliresponsive circularly polarized luminescence based on $\pi$-conjugated molecules. Pure Appl. Chem. 2013, 85, 1967-1978. [CrossRef]

9. Sanchez-Carnerero, E.M.; Agarrabeitia, A.R.; Moreno, F.; Maroto, B.L.; Muller, G.; Ortiz, M.J.; Moya, S. Circularly Polarized Luminescence from Simple Organic Molecules. Chem. Eur. J. 2015, 21, 13488-13500. [PubMed]

10. Kumar, J.; Nakashima, T.; Kawai, T. Circularly Polarized Luminescence in Chiral Molecules and Supramolecular Assemblies. J. Phys. Chem. Lett. 2015, 6, 3445-3452. [PubMed]

11. Longhi, G.; Castiglioni, E.; Kosyoubu, J.; Mazzeo, G.; Sergio, A. Circularly Polarized Luminescence: A Review of Experimental and Theoretical Aspects. Chirality 2016, 28, 696-707. [CrossRef]

12. Sun, Z.; Suenaga, T.; Sarkar, P.; Sato, S.; Kotani, M.; Isobe, H. Stereoisomerism, crystal structures, and dynamics of belt-shaped cyclonaphthylenes. Proc. Natl. Acad. Sci. USA 2016, 113, 8109-8114. [CrossRef]

13. Tanaka, H.; Inoue, Y.; Mori, T. Circularly Polarized Luminescence and Circular Dichroisms in Small Organic Molecules: Correlation between Excitation and Emission Dissymmetry Factors. ChemPhotoChem 2018, 2, 386-402. [CrossRef]

14. Pop, F.; Zigon, N.; Avarvari, N. Main-Group-Based Electro- and Photoactive Chiral Materials. Chem. Rev. 2019, 119, 8435-8478. [CrossRef] [PubMed]

15. Ma, J.-L.; Peng, Q.; Zhao, C.-H. Circularly Polarized Luminescence Switching in Small Organic Molecules. Chem. Eur. J. 2019, 25, 15441-15454. [CrossRef] [PubMed]

16. Ohishi, Y.; Inouye, M. Circularly polarized luminescence from pyrene excimers. Tetrahedron Lett. 2019, 60, 151232. [CrossRef]

17. Gao, J.; Zhang, W.Y.; Wu, Z.G.; Zheng, Y.X.; Fu, D.W. Enantiomorphic Perovskite Ferroelectrics with Circularly Polarized Luminescence. J. Am. Chem. Soc. 2020, 142, 4756-4761. [CrossRef] [PubMed]

18. He, C.; Feng, Z.; Shan, S.; Wang, M.; Chen, X.; Zou, G. Highly enantioselective photo-polymerization enhanced by chiral nanoparticles and in situ photopatterning of chirality. Nat. Commun. 2020, 11, 1188. [CrossRef]

19. Jin, Q.; Chen, S.; Sang, Y.; Guo, H.; Dong, S.; Han, J.; Chen, W.; Yang, X.; Li, F.; Duan, P. Circularly polarized luminescence of achiral open-shell $\pi$-radicals. Chem. Commun. 2019, 55, 6583-6586. [CrossRef] 
20. Zhao, J.; Zhang, T.; Dong, X.-Y.; Sun, M.-E.; Zhang, C.; Li, X.; Zhao, Y.S.; Zang, S.-Q. Circularly Polarized Luminescence from Achiral Single Crystals of Hybrid Manganese Halides. J. Am. Chem. Soc. 2019, 141, 15755-15760. [CrossRef]

21. Mason, S. Biomolecular homochirality. Chem. Soc. Rev. 1988, 17, 347-359. [CrossRef]

22. Girard, C.; Kagan, H.B. Nonlinear Effects in Asymmetric Synthesis and Stereoselective Reactions: Ten Years of Investigation. Angew. Chem. Int. Ed. 1998, 37, 2923-2959. [CrossRef]

23. Feringa, B.L.; Delden, R.A. Absolute Asymmetric Synthesis: The Origin, Control, and Amplification of Chirality. Angew. Chem. Int. Ed. 1999, 38, 3418-3438. [CrossRef]

24. Green, M.M.; Park, J.-W.; Sato, T.; Teramoto, A.; Lifson, S.; Selinger, R.L.B.; Selinger, J.V. The Macromolecular Route to Chiral Amplification. Angew. Chem. Int. Ed. 1999, 38, 3139-3154. [CrossRef]

25. Eschenmoser, A. Chemical Etiology of Nucleic Acid Structure. Science 1999, 284, 2118-2124. [CrossRef]

26. Soai, K.; Osanai, S.; Kadowaki, K.; Yonekubo, S.; Shibata, T.; Sato, I. $d$ - and l-Quartz-Promoted Highly Enantioselective Synthesis of a Chiral Organic Compound. J. Am. Chem. Soc. 1999, 121, 11235-11236. [CrossRef]

27. Sato, I.; Kadowaki, K.; Soai, K. Asymmetric synthesis of an organic compound with high enantiomeric excess induced by inorganic ionic sodium chlorate. Angew. Chem. Int. Ed. 2000, 39, 1510-1512. [CrossRef]

28. Kondepudi, D.K.; Asakura, K. Chiral Autocatalysis, Spontaneous Symmetry Breaking, and Stochastic Behavior. Acc. Chem. Res. 2001, 34, 946-954. [CrossRef]

29. Zepik, H.; Shavit, E.; Tang, M.; Jensen, T.R.; Kjaer, K.; Bolbach, G.; Leiserowitz, L.; Weissbuch, I.; Lahav, M. Chiral amplification of oligopeptides in two-dimensional crystalline self-assemblies on water. Science 2002, 295, 1266-1269. [CrossRef]

30. Sato, I.; Kadowaki, K.; Ohgo, Y.; Soai, K. Highly enantioselective asymmetric autocatalysis induced by chiral ionic crystals of sodium chlorate and sodium bromate. J. Mol. Catal. A Chem. 2004, 216, 209-214. [CrossRef]

31. Imai, Y.; Kamon, K.; Murata, K.; Harada, T.; Nakano, Y.; Sato, T.; Fujiki, M.; Kuroda, R.; Matsubara, Y. Preparation of a spontaneous resolution chiral fluorescent system using 2-anthracenecarboxylic acid. Org. Biomol. Chem. 2008, 6, 3471-3475. [CrossRef] [PubMed]

32. Imai, Y.; Murata, K.; Asano, N.; Nakano, Y.; Kawaguchi, K.; Harada, T.; Sato, T.; Fujiki, M.; Kuroda, R.; Matsubara, Y. Selective Formation and Optical Property of a 21-Helical Columnar Fluorophore Composed of Achiral 2-Anthracenecarboxylic Acid and Benzylamine. Cryst. Growth Des. 2008, 8, 3376-3379. [CrossRef]

33. Rexwinkel, R.B.; Meskers, S.C.J.; Riel, J.P.; Dekkers, H.P.J.M. Analysis of enantioselective quenching of tris(2,6-pyridinedicarboxylate)terbate(3-) luminescence by resolved tris(1,10-phenanthroline)ruthenium(2+) in methanol and in water. J. Phys. Chem. 1992, 96, 1112-1120. [CrossRef]

34. Petoud, S.; Muller, G.; Moore, E.G.; Xu, J.; Sokolnicki, J.; Riehl, J.P.; Le, U.N.; Cohen, S.M.; Raymond, K.N. Brilliant $\mathrm{Sm}, \mathrm{Eu}, \mathrm{Tb}$, and Dy Chiral Lanthanide Complexes with Strong Circularly Polarized Luminescence. J. Am. Chem. Soc. 2007, 129,77-83. [CrossRef] [PubMed]

35. Lunkley, J.L.; Shirotani, D.; Yamanari, K.; Kaizaki, S.; Muller, G. Extraordinary Circularly Polarized Luminescence Activity Exhibited by Cesium Tetrakis(3-heptafluoro-butylryl-(+)-camphorato) Eu(III) Complexes in EtOH and $\mathrm{CHCl}_{3}$ Solutions. J. Am. Chem. Soc. 2008, 130, 13814-13815. [CrossRef]

36. Walton, J.W.; Carr, R.; Evans, N.H.; Funk, A.M.; Kenwright, A.M.; Parker, D.; Yufit, D.S.; Botta, M.; Pinto, S.D.; Wong, K.-L. Isostructural Series of Nine-Coordinate Chiral Lanthanide Complexes Based on Triazacyclononane. Inorg. Chem. 2012, 51, 8042-8056. [CrossRef]

37. Zinna, F.; Bari, L.D. Lanthanide Circularly Polarized Luminescence: Bases and Applications. Chirality 2015, 27, 1-13. [CrossRef]

38. Zinna, F.; Giovanella, U.; Bari, L.D. Highly Circularly Polarized Electroluminescence from a Chiral Europium Complex. Adv. Mater. 2015, 27, 1791-1795. [CrossRef]

39. Richardson, F.; Brittain, H.G. A structural study of tris( $\beta$-diketonate)europium(III) complexes in solution using magnetic circularly polarized luminescence spectroscopy. J. Am. Chem. Soc. 1981, 103, 18-24. [CrossRef]

40. Foster, D.R.; Richardson, F.S. Magnetic circularly polarized luminescence of 9-coordinate europium(III) complexes in aqueous solution. Inorg. Chem. 1983, 22, 3996-4002. [CrossRef]

41. Foster, D.R.; Richardson, F.S.; Vallarino, L.M.; Shilladt, D. Magnetic circularly polarized luminescence spectra of $\mathrm{Eu}\left(\beta\right.$-diketonate) ${ }_{3} \mathrm{X}_{2}$ complexes in nonaqueous solution. Inorg. Chem. 1983, 22, 4002-4009. [CrossRef] 
42. Glover-Fischer, D.P.; Metcalf, D.H.; Hopkins, T.A.; Pugh, V.J.; Chisdes, S.J.; Kankare, J.; Richardson, F.S. Excited-State Enantiomer Interconversion Kinetics Probed by Time-Resolved Chiroptical Luminescence Spectroscopy. The Solvent and Temperature Dependence of $\Lambda$-Eu(dpa $)_{3}{ }^{3-} \rightleftarrows \Delta$-Eu(dpa $)_{3}{ }^{3-}$ Enantiomer Interconversion Rates in Solution. Inorg. Chem. 1998, 37, 3026-3033. [CrossRef]

43. Okutani, K.; Nozaki, K.; Iwamura, M. Specific Chiral Sensing of Amino Acids Using Induced Circularly Polarized Luminescence of Bis(diimine)dicarboxylic Acid Europium(III) Complexes. Inorg. Chem. 2014, 53, 5527-5537. [CrossRef]

44. Nelson, H.D.; Hinterding, S.O.M.; Fainblat, R.; Creutz, S.E.; Li, X.; Gamelin, D.R. Mid-Gap States and Normal vs Inverted Bonding in Luminescent $\mathrm{Cu}^{+}$- and $\mathrm{Ag}^{+}$-Doped CdSe Nanocrystals. J. Am. Chem. Soc. 2017, 139, 6411-6421. [CrossRef]

45. Jalilah, A.J.; Asanoma, F.; Fujiki, M. Unveiling controlled breaking of the mirror symmetry of Eu(fod $)_{3}$ with $\alpha$-/ $\beta$-pinene and BINAP by circularly polarised luminescence (CPL), CPL excitation, and ${ }^{19} \mathrm{~F}-\beta{ }^{1} \mathrm{P}\left\{{ }^{1} \mathrm{H}\right\}-\mathrm{NMR}$ spectra and Mulliken charges. Inorg. Chem. Front. 2018, 5, 2718-2733. [CrossRef]

46. Okada, H.; Hara, N.; Kaji, D.; Shizuma, M.; Fujuiki, M.; Imai, Y. Excimer-origin CPL vs monomer-origin magnetic CPL in photo-excited chiral binaphthyl-ester-pyrenes: Critical role of ester direction. Phys. Chem. Chem. Phys. 2020, 22, 13862-13866. [CrossRef] [PubMed]

47. Yoshikawa, H.; Nakajima, G.; Mimura, Y.; Kimoto, T.; Kondo, S.; Suzuki, A.; Fujiki, M.; Imai, Y. Mirror-image magnetic circularly polarized luminescence (MCPL) from optically inactive $\mathrm{Eu}^{\mathrm{III}}$ and $\mathrm{Tb}^{\mathrm{III}}$ tris( $\beta$-diketonate). Dalton Trans. 2020, 49, 9588-9594. [CrossRef]

48. Kaji, D.; Okada, H.; Hara, N.; Kondo, Y.; Suzuki, S.; Miyasaka, M.; Fujiki, M.; Imai, Y. Non-classically Controlled Sign in a 1.6 Tesla Magnetic Circularly Polarized Luminescence of Three Pyrenes in a Chloroform and a PMMA Film. Chem. Lett. 2020, 49, 674-676. [CrossRef]

49. Mislow, K.; Bickart, P. An epistemological note on chirality. Isr. J. Chem. 1977, 15, 1-6. [CrossRef]

50. Mislow, K. Absolute asymmetric synthesis: A commentary. Collect. Czech. Chem. Commun. 2003, 68, 849-864.

51. Amako, T.; Nakabayashi, K.; Suzuki, N.; Guo, S.; Rahim, N.A.A.; Harada, T.; Fujiki, M.; Imai, Y. Pyrene magic: Chiroptical enciphering and deciphering 1,3-dioxolane bearing two wirepullings to drive two remote pyrenes. Chem. Commun. 2015, 51, 8237-8240. [CrossRef] [PubMed]

52. Kimoto, T.; Tajima, N.; Fujiki, M.; Imai, Y. Control of Circularly Polarized Luminescence by Using Openand Closed-Type Binaphthyl Derivatives with the Same Axial Chirality. Chem. Asian J. 2012, 7, 2836-2841. [CrossRef] [PubMed]

53. Hara, N.; Yanai, M.; Kaji, D.; Shizuma, M.; Tajima, N.; Fujiki, M.; Imai, Y. A Pivotal Biaryl Rotamer Bearing Two Floppy Pyrenes that Exhibits Cryptochiral Characteristics in the Ground State. ChemistrySelect 2018, 3, 9970-9973. [CrossRef]

Publisher's Note: MDPI stays neutral with regard to jurisdictional claims in published maps and institutional affiliations.

(C) 2020 by the author. Licensee MDPI, Basel, Switzerland. This article is an open access article distributed under the terms and conditions of the Creative Commons Attribution (CC BY) license (http://creativecommons.org/licenses/by/4.0/). 